\title{
Modélisation des états de mer pour la recherche de sites d'exploitation houlomotrice Application à l'île de la Réunion
}

\author{
Jean Christophe Ascione ${ }^{1}$, Pierre Gaufrès ${ }^{2}$ \\ ${ }^{1}$ BCEOM - 78, Allée John Napier CS 89017, 34965 Montpellier \\ Cédex 2, France.jc.ascione@bceom.fr \\ ${ }^{2}$ CETMEF - Centre d'Etudes Techniques Maritimes Et Fluviales \\ Bd du Président Kennedy, B.P. 543, 13092 Aix-en-Provence \\ Cedex02, France.pierre.gaufres@equipement.gouv.fr
}

\section{Résumé :}

Une modélisation numérique des états de mer sur le littoral de la Réunion réalisée avec le logiciel Tomawac (plateforme Telemac) permet de caractériser les houles sur la côte Sud et évaluer le potentiel énergétique pour l'implantation d'usines houlomotrices. L'analyse est menée pour trois systèmes d'agitation qui cohabitent au large: houles d'alizés, australes et cycloniques. Trois types de maillage sont testés selon la bathymétrie des secteurs étudiés pour définir le maillage optimum, offrant les meilleures performances en terme de précision et de temps de calcul. Les résultats des simulations, croisés avec l'étude des zones d'intérêt remarquable, aboutissent à la localisation de secteurs à forts potentiels dans des profondeurs supérieures à 15 mètres, susceptibles d'accueillir des usines houlomotrices, compte tenu des impacts environnementaux et socio-économiques.

\section{Mots clefs :}

Énergie des vagues, analyse statistique, modélisation numérique de houle, maillage, réfraction, île de la Réunion.

\section{Introduction}

Dans un contexte de crise énergétique sur l'île, le Conseil Régional de la Réunion a décidé d'analyser le potentiel d'énergie renouvelable de l'océan compte tenu de la présence de houles puissantes et fréquentes qui balayent la zone. Les technologies de conversion de l'énergie des vagues en électricité se multiplient et arrivent à un stade proche de la commercialisation (DTI, $2003^{1}$ ), ainsi dans l'objectif d'exploiter ce type d'installation sur son territoire, la Région Réunion a lancé une étude visant à évaluer la ressource locale et à localiser les emplacements à fort potentiel pour y implanter des usines houlomotrices.

DOI: 10.5150/jngcgc.2006.002-A $\quad$ (disponible en ligne - http://www.paralia.fr - available online) 


\subsection{Présentation du site de l'étude}

La Réunion est une île volcanique et montagneuse située dans l'hémisphère Sud, entre l'Équateur et le tropique du Capricorne. Elle fait partie, avec les îles Maurice et Rodrigues, de l'archipel des Mascareignes. Bordée par l'Océan Indien, ses côtes d'une longueur totale de 207 kilomètres dont 40 kilomètres de plage, sont soumises à trois principaux types de houle : les houles australes, les houles d'alizés et les houles cycloniques.

\subsection{Objectif de l'étude}

A partir des données bathymétriques mises à disposition par le Conseil Régional de la Réunion et d'une analyse statistique des houles au large, une modélisation numérique des états de mer sur le littoral de la Réunion a été réalisée afin de caractériser les houles aux abords de la côte Sud. L'analyse des résultats de ces simulations a abouti à la localisation de zones à forts potentiels énergétiques susceptibles d'accueillir des usines houlomotrices (Soerensen H.C.et al., $2001^{2}$ ). Enfin, une analyse multicritères a permis de hiérarchiser les sites les plus propices à la réalisation d'un tel projet.

\subsection{Méthodologie}

La méthodologie de l'étude a consisté tout d'abord en un recueil de données qui a permis de définir les conditions hydrométéorologiques, la bathymétrie des fonds, les zones remarquables. L'étude des conditions météo-océaniques est essentielle non seulement pour réaliser une bonne modélisation des transferts de houles du large à la cote (conditions fréquentes), mais aussi pour définir les conditions de fréquence rare pouvant survenir sur le littoral. Ces conditions, dites extrêmes, permettront de préciser les contraintes d'utilisation et de dimensionnement des systèmes de récupération de l'énergie des vagues.

La bathymétrie autour de l'île a été étudiée finement car elle est essentielle au modèle de transfert des houles mais également à l'étude de faisabilité du projet. En effet, la profondeur est un critère déterminant pour la localisation d'une usine houlomotrice offshore; l'installation d'usines dans des zones trop profondes engendre des surcoûts d'ancrage importants qui peuvent remettre en question l'économie du projet.

Certaines zones remarquables ont été plus particulièrement étudiées : les zones portuaires, les espaces de pratique de sports nautiques (plaisance, plongée, etc....), les zones protégées (ZNIEFF, ZICO, Natura 2000, récifs coralliens, lagons, ...). 


\section{Modélisation de la propagation des houles}

\section{1. Étendue du modèle}

Le modèle de plan de vague doit avoir une emprise suffisante vers le large pour représenter au mieux la propagation des houles à l'approche des côtes Est et Ouest. Le logiciel utilisé pour modéliser le transfert des houles du large à la cote est le logiciel TOMAWAC de la plate forme TELEMAC développé par EDFLNHE. Le modèle aux éléments finis permet ainsi de définir les zones de concentration et de diffusion d'énergie et donc les hauteurs de houle à la côte. Les données bathymétriques utilisées ont été fournies par le SHOM (relevés 2002). Les points de sonde bathymétriques au large de la Réunion sont repérés dans le référentiel de projection UTM Sud fuseau 40 sur IAG GRS 80.

\subsection{Conditions de houle au large}

Les caractéristiques des houles au large représentent les conditions d'entrée du modèle et sont issues de l'analyse statistique réalisée lors de l'étude. Le tableau 1 présente les caractéristiques des houles modélisées.

\begin{tabular}{|l|c|c|c|}
\hline Mois & $\begin{array}{c}\text { angle/Nord } \\
\left({ }^{\circ}\right)\end{array}$ & $\begin{array}{c}\text { Hauteur } \mathbf{H}_{\mathbf{s}} \\
\mathbf{( m )}\end{array}$ & $\begin{array}{c}\text { Période T } \\
(\mathbf{s})\end{array}$ \\
\hline Janvier & 146 & 1.8 & 9 \\
\hline Février & 148 & 2 & 10 \\
\hline Mars & 155 & 2 & 10 \\
\hline Avril & 155 & 1.2 & 10 \\
\hline Mai & 159 & 2.2 & 10 \\
\hline Juin & 167 & 2.4 & 11 \\
\hline Juillet & 154 & 2.5 & 11 \\
\hline Août & 150 & 2.4 & 10 \\
\hline Septembre & 162 & 2.2 & 10 \\
\hline Octobre & 155 & 2 & 10 \\
\hline Novembre & 163 & 1.8 & 10 \\
\hline Décembre & 159 & 1.7 & 9 \\
\hline Moyenne annuelle & $\mathbf{1 5 6}$ & $\mathbf{2 . 1}$ & $\mathbf{1 0}$ \\
\hline Essai supplémentaire & 191 & 2.13 & 10.7 \\
\hline
\end{tabular}

Tableau 1 : Caractéristiques des houles modélisées sous Tomawac 


\subsection{Maillage}

La modélisation des plans de vague sur la côte Sud de l'île de la Réunion nécessite un maillage très fin et donc un nombre de nœuds de maille important afin d'assurer la convergence des calculs et la qualité des résultats.

La conception du maillage présente deux principales contraintes:

- être suffisamment fin dans les zones de faible profondeur où la réfraction des houles commence à apparaître,

- $\quad$ être suffisamment large afin de limiter le temps de calcul.

Afin de répondre à ces contraintes, la zone à modéliser a été divisée en trois modèles de taille sensiblement identique qui se recouvrent dans le but de se prémunir d'erreurs de calcul apparaissant à proximité des frontières du modèle (effets de bord): un modèle Ouest, un modèle Sud et un modèle Est. Le nombre de points de calcul varie de 20000 à 60000 pour chaque modèle et la longueur des mailles est comprise entre 20 et 2000 mètres au large (Figure 1).

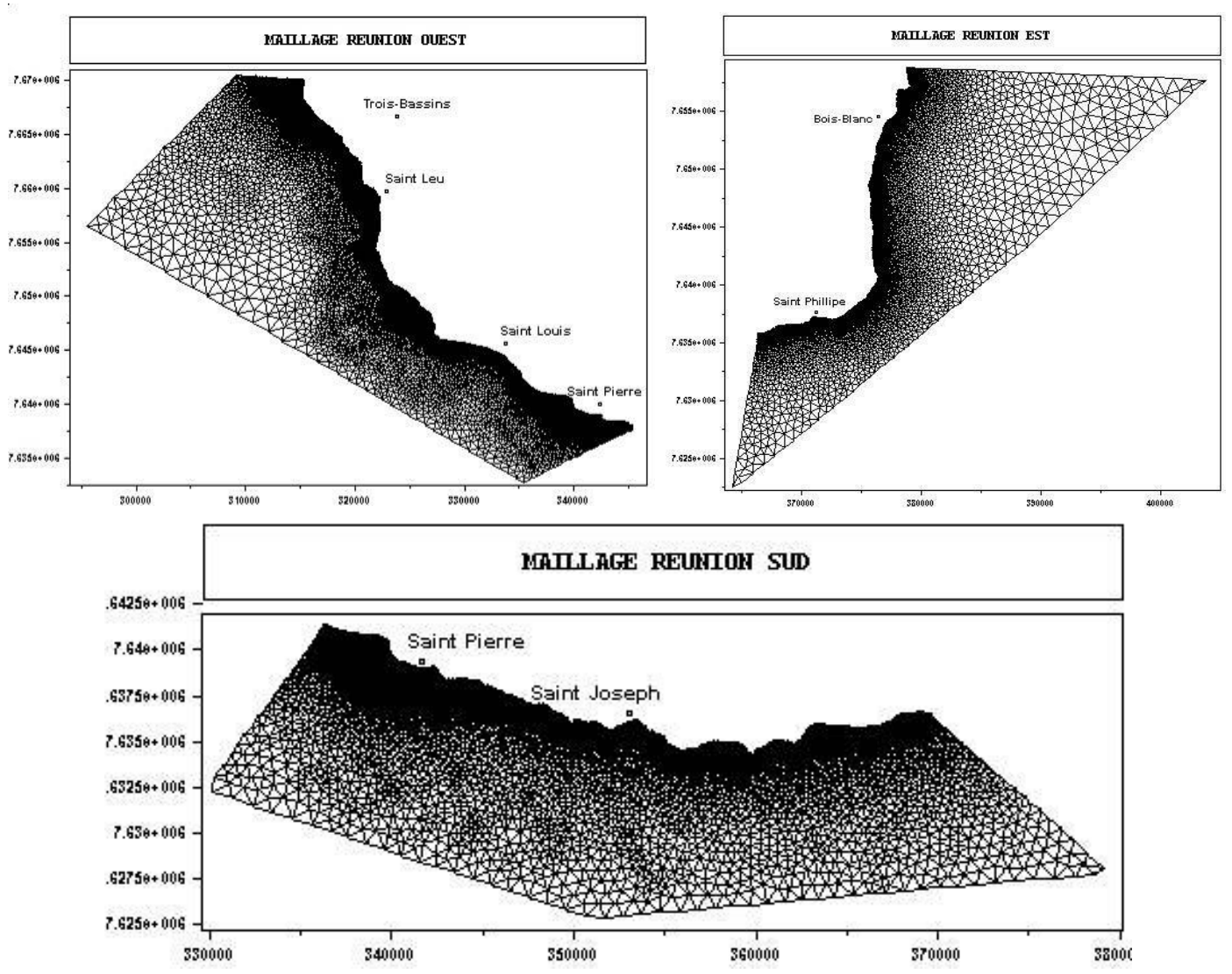

Figure 1: Emprise des modèles et maillages Réunion Ouest, Sud et Est

A partir d'une certaine profondeur, les processus de réflexion et de diffraction de la houle ne peuvent plus être négligés, les limites de validité du modèle sont alors 
atteintes. Dans le cadre de l'étude, le domaine où les résultats ne peuvent plus être considérés comme valides est localisé sur une bande étroite entre le trait de côte et la profondeur limite de validité, entre 12 et 15 mètres selon les conditions de houle modélisées. Cette profondeur limite n'est pas gênante compte tenu de la localisation usuelle des usines houlomotrices, dans des fonds variant de 25 à 100 mètres, exception faite des usines onshore.

Pour étudier plus précisément un site onshore, un modèle d'agitation prenant en compte la diffraction et la réflexion devra être réalisé (Thorpe T.W., $1999^{3}$ ), il pourra être couplé au modèle de réfraction.

\subsection{Résultats}

Les simulations obtenues avec le logiciel TOMAWAC ont permis de calculer la période de la houle, la hauteur moyenne des vagues ainsi que la direction de propagation de la houle par rapport au nord pour les conditions initiales précitées (Peronno G., $2003^{4}$ ). La théorie de la houle (Bonnefille R., $1992^{5}$ ) établit que la période reste constante lors sa propagation hors zone de déferlement. Ainsi le potentiel énergétique, exprimé en kilowatt par mètre, peut être calculé à partir de la formule suivante :

$$
P=\frac{1}{16} \rho_{\text {eau }} g H_{m}^{2} C
$$

avec :

- $\quad \mathrm{P}$ : potentiel énergétique de la houle $\left(\mathrm{kW} \cdot \mathrm{m}^{-1}\right)$

- $\quad \rho_{\text {eau }}$ : masse volumique de l'eau de mer $\left(1025 \mathrm{~kg} \cdot \mathrm{m}^{-3}\right)$

- C : célérité de la houle au large et pour une profondeur donnée $\left(\mathrm{m} \mathrm{s}^{-1}\right)$

- $\mathrm{H}_{\mathrm{m}}$ : hauteur moyenne de la houle définie par l'étude statistique (m)

- $\mathrm{g}$ : accélération de la pesanteur $\left(9.81 \mathrm{~m} . \mathrm{s}^{-2}\right)$

Après analyse spectrale sur une chronique de 10 années (1992-2002), les houles retenues ne prennent pas en compte les vents d'alizés en zone littorale qui sont susceptibles d'amplifier de façon non négligeable les houles mesurées au large et ainsi d'augmenter le potentiel énergétique des sites, au Sud et au Sud-Est de la zone d'étude notamment. Les résultats de répartition énergétique montrent une faible variation de l'énergie potentielle, même à l'approche des côtes. Les phénomènes de réfraction sont sensibles à partir de profondeurs égales au tiers de la longueur d'onde des houles arrivant sur le site. Les houles retenues de longueur d'onde de l'ordre de 190 mètres commencent à réfracter pour des profondeurs de 60 mètres environ. Or les données bathymétriques disponibles s'arrêtent à la profondeur de 30 mètres et le domaine de validité des modèles de réfraction est limité à 15 mètres pour ce type de houle. Ainsi, la localisation fine de sites littoraux (onshore) n'est pas réalisable à partir de ce type de modélisation, cependant il est possible d'identifier les zones de fort potentiel énergétique, ce qui permet de réduire l'étendue des modèles d'agitation nécessaires à la définition des sites littoraux. 


\section{Discussion}

\subsection{Méthodologie de sélection des sites à fort potentiel énergétique}

L'étude de la bathymétrie aux abords du littoral Sud de la Réunion fait apparaître que les fonds remontent très rapidement. Les houles n'ont donc pas le temps de subir les effets de la réfraction qui pourrait générer des zones de concentration d'énergie; ainsi de nombreux sites ont un potentiel énergétique équivalent.

La sélection des sites a été effectuée à partir de plusieurs critères (Previsic M., $2004^{6}$ ):

- potentiel énergétique supérieur ou égal à $95 \%$ du potentiel maximum,

- éloignement de la côte (entre 200 et 2000 mètres) et profondeur (inférieure à 150 mètres),

- proximité de zones d'intérêts particuliers (récifs coralliens, etc.).

- caractéristiques fonctionnelles du littoral,

- proximité d'installations portuaires ou de zones à fort besoin énergétique.

La proximité de zones à fort besoin énergétique est en effet un critère de sélection important pour la localisation des installations car elle permet de limiter les pertes dans le réseau et donc d'améliorer les rendements en puissance fournis aux usagers par les usines houlomotrices.

Six sites ont été ainsi retenus sur la base de ces critères :

- Quai d'Henri Dalleau,

- Pointe du bétail,

- Pointe Langevin,

- Pointe du Parc,

- Étang Salé (le gouffre),

- Saint-Leu (face à la ravine du Grand Étang).

La plupart sont des caps ou des avancées qui permettent de diminuer les distances entre les installations et le littoral.

Pour une houle de caractéristiques données (hauteur, direction, période, énergie), le potentiel énergétique de la moyenne annuelle est représenté (exemple Figure 2).

L'analyse des potentiels énergétiques révèle que les zones à fort potentiel sont systématiquement situées dans des zones de fortes profondeurs où les houles subissent moins les effets de la bathymétrie et perdent donc moins d'énergie. La modélisation des potentiels énergétiques confirme que la remontée des fonds est trop acore sur la zone d'étude pour que les houles puissent subir de façon importante les effets de la réfraction sur le fond. 


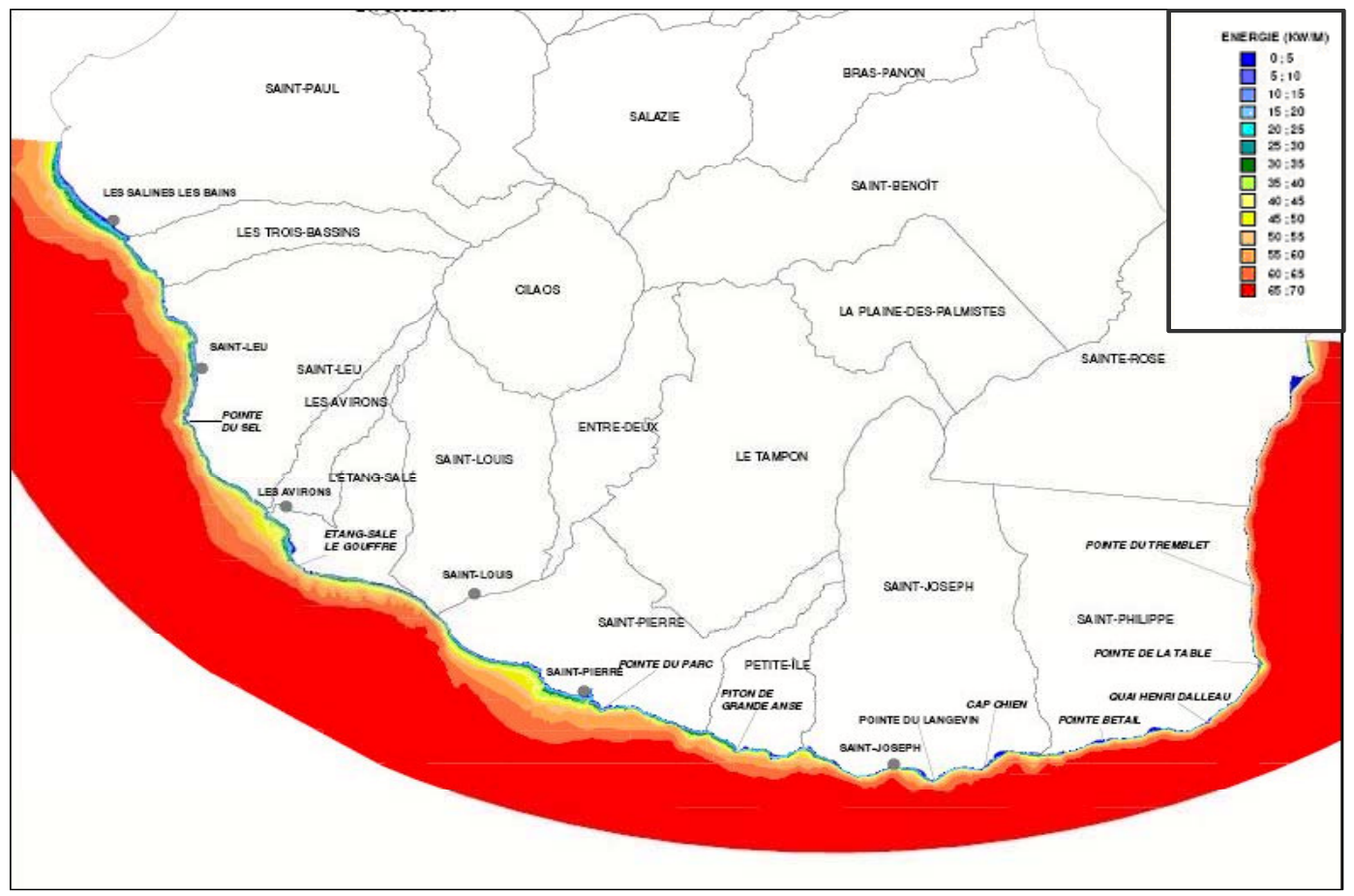

Figure 2 : Réunion Sud - Représentation de l'énergie $(\mathrm{kw} / \mathrm{m})-\mathrm{H}_{\mathrm{s}}=2,5 \mathrm{~m} \mathrm{~T}_{\mathrm{p}}=11 \mathrm{~s}$

\subsection{Analyse multicritères}

L'analyse comparative des sites au plan environnemental s'appuie sur une comparaison des impacts paysagers, écologiques, sédimentaires et sonores. Un autre critère de comparaison retenu est le critère socio-économique qui prend en compte la localisation géographique, les ressources socio-économiques et un critère fonctionnel (installation, maintenance, ...). Dans un premier temps, six sites de potentiel énergétique comparable ont été retenus sur la base des critères généraux évoqués précédemment.

\section{Conclusion}

L'identification de six zones à fort potentiel énergétique sur la cote Sud de la Réunion a été mise en évidence à partir de simulations numériques sous TOMAWAC. La modélisation des plans de vague a nécessité un maillage très fin jusqu'aux fonds de 15 mètres à proximité des cotes et permis de réduire l'étendue des modèles d'agitation nécessaires à la définition des projets sur les sites littoraux (onshore). L'analyse multicritères distingue le site de la Pointe du Parc sur la partie Est de la Commune de Saint-Pierre (70 000 habitants), à 1250 mètres de la cote, comme situation optimale. Ce site est à la fois à proximité d'infrastructures portuaires importantes et de forts besoins énergétiques, conditions essentielles au projet. Les sites de Saint-Leu et de la Pointe Langevin présentent aussi des aspects 
intéressants pour l'installation d'une usine houlomotrice. Un relevé bathymétrique précis des zones identifiées permettra d'optimiser l'implantation des installations houlomotrices en fonction du potentiel énergétique local par modélisation des phénomènes de diffraction notamment dans les études d'agitation.

\section{Bibliographie}

1 DTI, (2003), Wave and marine current energy, International Energy Agency, report No FES-R-132

2 Soerensen H.C., Hansen R., (2001), Low-pressure Hydro Turbines and Control Equipment for Wave Energy Converters (Wave Dragon), EU Publishable Final Report.

3 Thorpe T.W., (1999), A brief review of wave energy, Department of Trade and Industry, UK, Report No. ETSU-R-120.

4 Peronno G., (2003), Énergie des vagues - île de la Réunion. ARER.

5 Bonnefille R., (1992), Cours d'hydraulique maritime, troisième édition.

6 Previsic M., (2004), Assessment offshore wave energy conversion devices, E2I EPRI WP -004-US-REV1.

\section{6. $\quad$ Remerciements}

Les auteurs remercient le Conseil Régional de la Réunion pour l'aimable autorisation à publier les résultats de l'étude et les services de Météo France pour la fourniture des données de forçages météomarins au large. 